\title{
In memoriam Dr. Sabine Rüsch-Gerdes
}

Dieser Nachruf soll Dr. Sabine RüschGerdes ehren, die am 11. Juli 2017 im Alter von 68 Jahren verstorben ist. In Deutschland, aber auch international, hat sie die Mykobakterien-Diagnostik nachhaltig geprägt und wird sehr vielen Menschen als engagierte Persönlichkeit mit grenzenloser Energie in Erinnerung bleiben.

Die mykobakteriologische Laufbahn von Sabine Rüsch-Gerdes begann nach der Doktorarbeit an der Universität Hamburg als stellvertretende Leitung in der Mikrobiologie des damaligen Forschungsinstituts Borstel, in der sie ab 1993 die Leitung übernommen hat. Von Beginn an hat sie sich für die Verbesserung und Modernisierung der mikrobiologischen Verfahren in der Tuberkuloseund Mykobakterien-Diagnostik engagiert, immer mit dem Anspruch qualitativ hochwertiger Arbeit. Nach der Einführung neuer und verlässlicher Methoden im eigenen Labor war ihr der Wissenstransfer zur Weiterentwicklung auch anderer Laboratorien ein hohes Anliegen. Frühzeitig hat sie dabei erkannt, dass vor allem in der Unterstützung zum Aufbau und zur Weiterentwicklung von Laboratorien in „Hoch-Inzidenz“-Ländern ein entscheidender Beitrag zu einer weltweiten Verbesserung der TB-Situation liegt.

Ihr herausragendes Engagement und das hohe Niveau der Diagnostik führten 1990 zur Ernennung des Borsteler Labors zum Nationalen Referenzzentrum für Mykobakterien unter der Leitung von Sabine Rüsch-Gerdes, welches sie bis zu ihrem Ausscheiden 2014 weiterführte. Sie hat in dieser Zeit die Diagnostik der Tuberkulose in den deutschsprachigen Ländern mit Leidenschaft und Engagement nachhaltig beeinflusst durch zahllose Vorträge, Veröffentlichungen, Buchbeiträge und Aktivitäten im Arbeitskreis Mykobakterien oder dem DIN Normen- ausschuss „TB-Diagnostik“, aber auch ganz praktisch mit hilfreichen und lebhaften Telefonaten oder unmittelbarer Hilfe. Viele Gäste des NRZ konnten sich direkt vor Ort die Laborabläufe aneignen, mit den Prozessen im eigenen Labor vergleichen und uneingeschränkt Fragen stellen. Nahezu alle neuen Methoden, die die Diagnostik beschleunigen und verbessern sollten, wurden in ihrem Labor evaluiert und ehrlich bewertet. Dies führte oftmals zu Verbesserungen der noch in der Entwicklung befindlichen Verfahren. Das Kriterium, „wurde im NRZ evaluiert“, galt als Qualitätsmerkmal. Die für die Laboratorien verpflichtend durchzuführenden TB-Ringversuche wurden seit einigen Jahren durch sie organisiert und über INSTAND e. V. nicht nur in Deutschland, sondern weltweit versendet und eingesetzt. Sabine RüschGerdes' beratende Unterstützung war auch für alle in der Tuberkulosefürsorge tätigen Mitarbeiter des Öffentlichen Gesundheitsdienstes jederzeit abrufbar. Überregionale Studien, wie z. B. Fingerprint-Studien, wären ohne ihr kollegiales und kooperatives Zusammenführen der vielen Partner nicht möglich gewesen. Jederzeit war sie ansprechbar für Probleme in den Laboratorien, unabhängig vom Anrufer. Ebenso war sie vielgefragte Ratgeberin für viele klinische Ärzte in Fragen zur Diagnostik und Therapie von Tuberkulose und Mykobakteriosen. Junge Ärzte und Wissenschaftler wurden von ihr ermuntert und unterstützt, sich für das Thema Tuberkulose zu interessieren und sich den Kampf gegen die Tuberkulose zum Ziel zu setzen.

Die internationalen Aktivitäten von Sabine Rüsch-Gerdes wurden 1995 durch die Ernennung zum Supranationalen Referenzlabor durch die WHO anerkannt und auf diesem Weg in ein internationales Aktionsbündnis eingefügt. Mit ihrem Enthusiasmus und ihrer unerschöpfli-

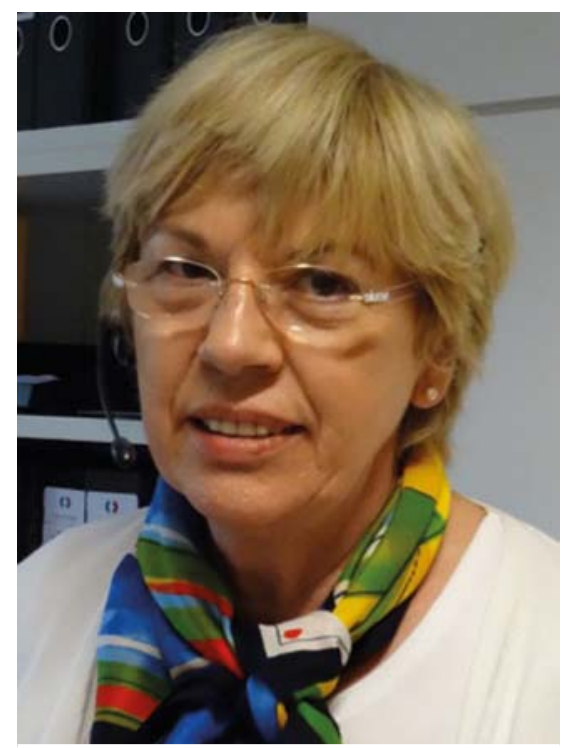

Dr. Sabine Rüsch-Gerdes.

chen Energie ist sie weltweit zu vielen TB-,Hotspots“ gereist und hat dabei viele Organisationen wie die WHO, MSF, KNCV, DGZ, ICRC oder FIND bei TB-Projekten unterstützt. Durch ihre Erfahrung und Sachkenntnis war sie eine vielgefragte Beraterin für nationale und internationale Gremien und Organisationen. Im Netzwerk der Supranationalen Referenzlabore entwickelte sie ihres zu einem der führenden. In vielen Ländern Zentralasiens und Afrikas hat sie die Laboratorien mit Verve, Wissen und Überzeugungskraft verbessert und sie in ihrer Position im Gesundheitssystem gestärkt.

Ihre wissenschaftlichen Verdienste wurden national und international durch die Verleihung des Bundesverdienstkreuzes und des „Gardner Middlebrook-Awards“ der „American Society for Microbiology“ gewürdigt.

Sabine Rüsch-Gerdes war stets bewusst, dass die von ihr angegangenen Aufgaben und Herausforderungen nur durch die 
Unterstützung engagierter Mitarbeiter bewältigt werden können. Ihr „Team“ war ihr persönlich wichtig, und sie hat jeden einzelnen als Menschen und in seiner Bedeutung für die vielzähligen Aktivitäten immer anerkannt.

Ihr gebührt große Anerkennung für ihr lebenslanges herausragendes persönliches Engagement, ihre Expertise auf vielen verschiedenen Ebenen einzusetzen mit dem Ziel, die Bedeutung verlässlicher TB-Diagnostik zu vermitteln. So waren politische Entscheidungsträger genauso ihre Ansprechpartner wie das technische Personal in unzähligen Laboratorien. Für diesen persönlichen Einsatz danken wir ihr.
Sabine Rüsch-Gerdes hat durch ihre Nähe zu den Menschen, ihre Geradlinigkeit, ihre humanitäre Grundhaltung, aber auch durch ihre Spontaneität eine Verbindung zu anderen Menschen aufbauen und viele dadurch motivieren können, auch schwierige Themen anzugehen und ein gemeinsames Ziel zu verfolgen. Sie wird uns allen durch ihren lebhaften, engagierten und mutigen Charakter in Erinnerung bleiben.

Wir trauern um einen großartigen Menschen, eine engagierte Mentorin und Kollegin, eine Freundin.
PD Dr. Elvira Richter, Heidelberg; Labor Limbach, TB-Labor, Für das DZK: Prof. Dr. Torsten Bauer, Berlin, Generalsekretär des DZK; Prof. Dr. Tom Schaberg, Rotenburg Für die DGP: Prof. Dr. Klaus Rabe, Großhansdorf, Präsident der DGP Für den AK Mykobakterien und den Normenausschuss „TB-Diagnostik“: Dr. Michael Klotz, Saarbrücken

\section{Korrespondenzadresse}

PD Dr. Elvira Richter

Leitung TB-Labor

MVZ Labor Dr. Limbach \& Kollegen GbR

Im Breitspiel 16

69126 Heidelberg

elvira.richter@labor-limbach.de 